\title{
Exit time for a reaction diffusion model: Case of a one well potential
}

\author{
Adrian Hinojosa ${ }^{\mathrm{a}}$ \\ ${ }^{\mathrm{a}}$ Federal University of Minas Gerais
}

\begin{abstract}
We consider a interacting particle system, the Glauber + Kawasaki model. This model is the result of the combination of a fast stirring, the Kawasaki part, and a spin flip process, the Glauber part. This process has a Reaction-Diffusion equation as hydrodynamic limit, as is proven by De Masi and Presutti (Mathematical Methods for Hydrodynamic Limits (1991) Springer). The ergodicity of these dynamics (one well potential) was proven in Brasseco et al. (Amer. Math. Soc. Transl. Ser. 2198 (2000) 37-49), for any dimension. In this article, we prove the asymptotic exponentiality for certain exit time from a subset of the basin of attraction of the well.
\end{abstract}

\section{Introduction}

The class of interacting particle systems that we study, was proposed by De Masi, Ferrari and Lebowitz, as alternative models for reaction-diffusion systems. On the configuration space $\{-1,+1\}^{\mathbb{Z}^{d}}$, they are obtained from the superposition of a Glauber (spin flip) type dynamics, corresponding to the reactive part, and a stirring one, also called Kawasaki dynamics at infinite temperature, that corresponds to the diffusive part, which is speeded by a factor, say $\varepsilon^{-2}$. The kinetic limit, which here corresponds to the hydrodynamical one, involves the simultaneous change of space scaling in the diffusive limit for the stirring, to provide the macro scale (macro $=$ $\varepsilon$ micro). Under such limit, the macroscopic description is verified, being given by a reaction diffusion equation of the form

$$
\partial_{t} m=\Delta m+F(m),
$$

where $m(r, t) \in \mathbb{R}$ represents the magnetization or density. The force term $F(\cdot)$ being determined by the Glauber rates. For example, given any polynomial $F(\cdot)$ we may choose finite range spin flip rates which lead to the above equation (of course there are innumerous choices, though the behaviour should be similar). That is, the empirical magnetization, or the density of particles in small boxes, converges in probability to the solution of the reaction diffusion equation. Indeed one knows more: strong forms of propagation of chaos (i.e., asymptotic independence of different spins) have been proven, initially by De Masi, Ferrari and Lebowitz (1986),

Key words and phrases. Exit times, interacting particle systems, Glauber-Kawasaki dynamics, reaction-diffusion equations, hydrodynamic limits.

Received August 2015; accepted April 2017. 
and later in sharper forms by several authors, see De Masi and Presutti (1991). At the level of propagation of chaos, more general systems, with $m(r, t)$ taking values in $\mathbb{R}^{n}$ are treated just in the same way, though we restrict ourselves to the case $n=1$. So there is no loss in assuming $F(m)=-V^{\prime}(m)$ in the above equation.

For a class of rates leading to a single well potential, the ergodicity for any $d \geq 1$, has been proven by Brassesco et al. (2000a). The same authors also proved ergodicity in the case of a double well potential, provided $V(\cdot)$ has a unique minimizer, cf. Brassesco et al. (2000b). We also refer to the article of Durrett and Neuhauser (1994), where a large classes of spin flip rates were considered, with the characteristic of having a trapping configuration (e.g. the contact process). In such case phase transition might occur, for the same reason which leads to the existence of an invariant measure with magnetization different from the trapping configuration, that is, is related to the minimizer of the potential.

The problem which we want to address refers to the large deviation behaviour of the process, fixing Glauber rates which lead to a one well potential. Our goal is to investigate the asymptotic loss of memory for the exit time from attracting sets contained in the "basin of attraction" of the ergodic measure. Connected to the reaction-diffusion model, related questions have been considered when the system is obtained by the addition of a small random noise to the equation (1.1), as treated by Martinelli, Olivieri and Scoppola (1989), Brassesco, Olivieri and Vares (1998), using coupling arguments, together with estimates of large deviations, which in this infinite dimensional setup were obtained by Faris and Jona-Lasinio (1982), extending Freidlin and Wentzell results. There are many analogous results in the context of low temperature Glauber dynamics on finite volumes, indeed extended to a large class of Markov chains, but they involve rather different methods.

We consider a macroscopically finite volume, that is, a torus of side $\varepsilon^{-1}$, though the result extends to larger volumes, which are tending to infinity in the macro scale, as for instance, $\varepsilon^{-l}, l>1$. Also, for simplicity we prove for dimension $d=1$, and study the problem when we start the process from the Bernoulli measure, which has the mean in one of the basins of the attraction. There is no problem to generalizing to dimensions $d \geq 1$, and to consider the initial configuration starting from an smooth profile, which is contained also in a basin of attraction. We prove that the properly rescaled exit time follows an exponential law. This result uses more simple ideas than in Hinojosa (2004), because, in the case of a one well potential, it is possible to couple two evolutions of the process starting in the same basin of attraction in a time of order $a|\log \varepsilon|$ with a probability of order $\varepsilon^{n}$, see Proposition 4.2 below, this fact was proven in Brassesco et al. (2000a). In the case of a double well potential, there exists a similar result, see Brassesco et al. (2000b), but the probability of this event is bounded by $c_{\varepsilon} \varepsilon^{n}$, where $c_{\varepsilon}$ is related to the passage from one well to the other, the exponential exit time result is still valid, see Hinojosa (2004), but the proof is more difficult. Finally, we use a result from Azlarov and Volodin (1986), which bound the uniform distance between $e^{-t}$ and $\mathbb{P}(\xi>t)$ ( $\xi$ is a random variable) from a bound over $\mathbb{P}(\xi>t+s \mid \xi>s)-\mathbb{P}(\xi>t)$; this is the loss of memory property. 


\section{Definitions and results}

We consider, for each $\varepsilon>0$, a family of Markov process $\left\{\sigma_{t}\right\}_{t \geq 0}, \sigma_{t} \in \Omega^{\varepsilon}=$ $\{-1,1\}^{\mathbb{Z}_{\varepsilon}}$, where we let $\mathbb{Z}_{\varepsilon}$ be the set of integers $\bmod \left[\varepsilon^{-1}\right],[x]$ is the integer part of $x \in \mathbb{R}: \mathbb{Z}_{\varepsilon}=\mathbb{Z}_{\left[\varepsilon^{-1}\right]}=\left\{0,1, \ldots,\left[\varepsilon^{-1}\right]\right\}$, with both ends identified. The evolution of the process is governed by the generator:

$$
L_{\mu}^{(\varepsilon)}=\varepsilon^{-2} L_{0}+L_{\mu}
$$

where $L_{0}$ is the generator of the stirring process:

$$
L_{0} f(\sigma)=\sum_{|x-y|=1}\left[f\left(\sigma^{x, y}\right)-f(\sigma)\right],
$$

$f$ is a function in $\Omega^{\varepsilon}, x, y \in \mathbb{Z}_{\varepsilon}$, and

$$
\sigma^{x, y}(z)= \begin{cases}\sigma(z), & z \neq x, y \\ \sigma(y), & z=x \\ \sigma(x), & z=y,\end{cases}
$$

and $L_{\mu}$ is the generator of the spin flip process:

$$
L_{\mu} f(\sigma)=\sum_{x \in \mathbb{Z}_{\varepsilon}} c_{\mu}(x, \sigma)\left[f\left(\sigma^{x}\right)-f(\sigma)\right],
$$

again $f$ is a function in $\Omega^{\varepsilon}$, and

$$
\sigma^{x}(z)= \begin{cases}-\sigma(z), & z=x \\ \sigma(z), & z \neq x\end{cases}
$$

$c_{\mu}(x, \sigma)$, the spin flip intensity, is:

$$
c_{\mu}(x, \sigma)=c_{0}(x, \sigma)-\frac{\mu}{2} \sigma(x),
$$

and $c_{0}(x, \sigma)$ is defined as:

$$
c_{0}(x, \sigma)=1-\gamma \sigma(x)[\sigma(x-1)+\sigma(x+1)]+\gamma^{2} \sigma(x-1) \sigma(x+1),
$$

$\mu$ is restricted to $0 \leq \mu<2(1-\gamma)^{2}$ for $c_{\mu}(x, \sigma)$ to be positive, we will consider in this article the case of $\mu=0$, and $\gamma \in\left(0, \frac{1}{2}\right)$, so $c_{\mu}(x, \sigma)=c_{0}(x, \sigma)$. The process defined above is called the Glauber + Kawasaki process.

If $v$ is a probability on $\Omega^{\varepsilon}$ (resp. a single configuration $\sigma$ ), we will denote by $E_{v}^{(\varepsilon)}$ (resp. by $E_{\sigma}^{(\varepsilon)}$ ) the expectation of the above process starting with law $v$ (resp. from the configuration $\sigma$ ); also we write $P_{\nu}^{(\varepsilon)}$ (resp. $P_{\sigma}^{(\varepsilon)}$ ) when we refer to the law of the process. In De Masi, Ferrari and Lebowitz (1986), it was proved that, as $\varepsilon \rightarrow 0$, the process, in infinite volume, converges to the solution of the reactiondiffusion equation:

$$
\frac{\partial m}{\partial t}=\Delta m-V_{\mu}^{\prime}(m)
$$



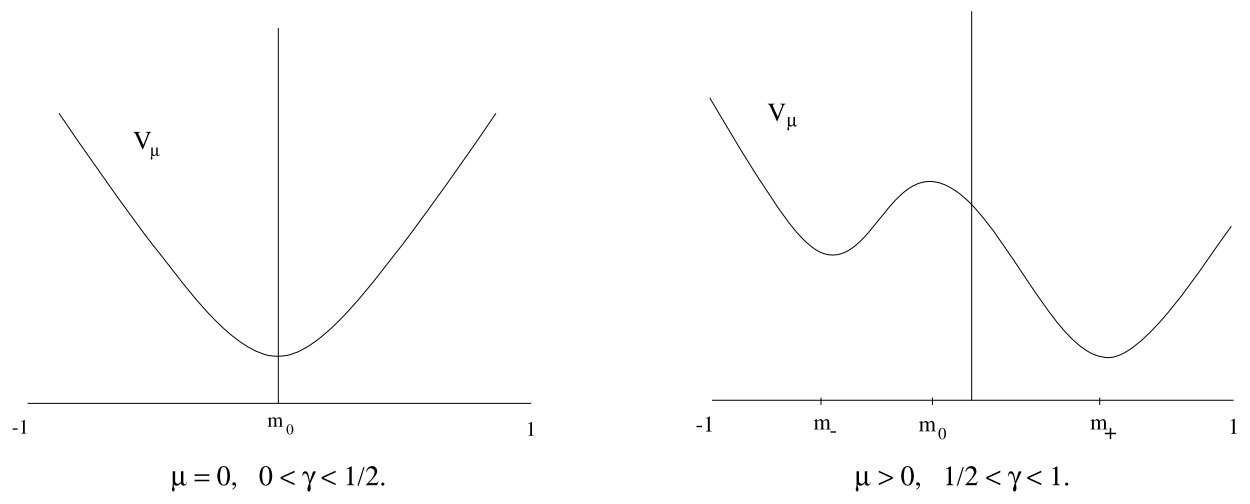

Figure 1 One well potential on the left. Two well potential on the right.

where:

$$
-V_{\mu}^{\prime}=\mathbb{E}_{v_{m}}\left(-2 \sigma(0) c_{\mu}(0, \sigma)\right)=-\alpha m-\beta m^{3}+\mu,
$$

$v_{m}$ denoting the Bernoulli product measure on $\Omega^{\varepsilon}$ with average $m$ and

$$
\alpha=2(1-2 \gamma), \quad \beta=2 \gamma^{2} \text {. }
$$

In the case $\mu=0$, the derivative of the potential is:

$$
-V^{\prime}=\mathbb{E}_{v_{m}}(-2 \sigma(0) c(0, \sigma))=-\alpha m-\beta m^{3} .
$$

The polynomial $V_{\mu}^{\prime}$ is the derivative of the, in general double well, potential $V_{\mu}$ (see Figure 1), and in the case we will consider $\left(\mu=0\right.$, and $\left.\gamma \in\left(0, \frac{1}{2}\right)\right)$ that potential $V$ has only one well, with a minimum at 0 .

The case of infinite volume (i.e., $\{-1,1\}^{\mathbb{Z}^{d}}$ ) was considered in Brassesco et al. (2000a) (one well) and in Brassesco et al. (2000b) (two wells), with the same dynamics. It was proved there that for $\varepsilon$ small enough the process is ergodic (in our case the process is immediately ergodic, since we are in finite volume). Observe that (2.6) is not ergodic (in the sense that it has two invariant solutions), also that $m=0$ is the minimum in the case that we consider.

We shall consider the set of configurations with empirical magnetization in small intervals (of size $\varepsilon^{-a}$, where $0<a<1$ is close to one), belongs to $[-\delta, \delta]$, with $\delta$ small enough, that is,

$$
A_{\delta}^{(\varepsilon)}=\left\{\sigma \in \Omega^{\varepsilon}:\left|\varepsilon^{a} \sum_{x:|x| \leq \frac{\varepsilon^{-a}}{2}} \sigma(x+y)\right|<\delta, \forall y \in \mathbb{Z}_{\varepsilon}\right\} .
$$

When it is clear from the context we drop the superscript $\varepsilon$ and write simply $A_{\delta}$. Now consider the Bernoulli product measure, $v_{m}$, on $\Omega^{\varepsilon}$ with average $m \in[-\delta, \delta]$, and define the following stopping time

$$
\tau^{\varepsilon}=\inf \left\{t>0: \sigma_{t} \notin A_{\delta}^{(\varepsilon)}\right\} .
$$


Also, define $\beta_{\varepsilon}$ as: $P_{v_{m}}^{(\varepsilon)}\left(\tau^{\varepsilon}>\beta_{\varepsilon}\right)=e^{-1}$. Regarding the asymptotic distribution of $\tau^{\varepsilon} / \beta_{\varepsilon}$, the main result is the following theorem.

Theorem 2.1. For $\tau^{\varepsilon}$ defined above and $m \in[-\delta, \delta]$ it holds:

$$
\lim _{\varepsilon \rightarrow 0} \sup _{t \geq 0}\left|P_{v_{m}}^{(\varepsilon)}\left(\tau^{\varepsilon}>t \beta_{\varepsilon}\right)-e^{-t}\right|=0
$$

\section{Proof of Theorem 2.1}

We shall prove that if we start the process from the Bernoulli product measure, $v_{m_{-}}$, then, under $P^{(\varepsilon)}, \tau^{\varepsilon} / \beta_{\varepsilon}$ converges, in the Levy metric, to a mean one exponential r.v. First, we prove that $\tau^{\varepsilon} / \beta_{\varepsilon}$ is uniformly close, in the Levy metric, to a family of exponentials r.v.; and then that this family converges also in the Levy metric to a mean one exponential r.v. As convergence in Levy metric, with an absolutely continuous limit, implies convergence in Kolmogorov metric, then the theorem is proved.

Let $k \geq 1$ be fixed. In Hinojosa (2004), it is proved the following lower bound for $\beta_{\varepsilon}$ : for any $n \geq 1$, there exists $c_{n}>0$, such that for $\varepsilon>0$ small enough it holds:

$$
P_{v_{m}}^{(\varepsilon)}\left(\tau^{\varepsilon}<\varepsilon^{-k}\right) \leq c_{n} \varepsilon^{n}
$$

Note that, by the Cramer-Chernov theorem for large deviations for i.i.d. (Bernoulli) random variables, for any $\delta^{\prime}>0$, at time zero

$$
\begin{aligned}
P_{v_{m}}^{(\varepsilon)}\left(A_{\delta^{\prime}}^{c}\right) & \leq \varepsilon^{-1} P_{v_{m}}^{(\varepsilon)}\left(\left|\varepsilon^{a} \sum_{|x| \leq \frac{\varepsilon^{-a}}{2}} \sigma(x)\right| \geq \delta^{\prime}\right) \\
& \leq \varepsilon^{-1} e^{-\tilde{c} \varepsilon^{-a}} \leq e^{-c \varepsilon^{-a}} .
\end{aligned}
$$

For some $c>0$. Then we shall consider that with large $v_{m}$-probability the initial configuration starts from $A_{\delta^{\prime}}$, for any $\delta^{\prime}<\delta$. Also, since (3.1), if $\varepsilon$ is small enough we have that:

$$
P_{v_{m}}^{(\varepsilon)}\left(\tau^{\varepsilon}>\varepsilon^{-k}\right)>e^{-1}
$$

But by definition $P_{v_{m}}^{(\varepsilon)}\left(\tau^{\varepsilon}>\beta_{\varepsilon}\right)=e^{-1}$, therefore $\varepsilon^{-k}<\beta_{\varepsilon}$, for any $k \geq 1$, and $\varepsilon$ small enough.

The main part of the proof is contained in Proposition 3.1. It says that the family of r.v. $\tau^{\varepsilon} / \beta_{\varepsilon}$ has, uniformly in $\varepsilon$, loss of memory property (note that this feature characterizes the exponential distribution). From this proposition, we shall prove that, in fact, for each $\varepsilon$ we have an exponential random variable that is close, in the Levy metric sense, to $\tau^{\varepsilon} / \beta_{\varepsilon}$.

Before stating the loss of memory property, we let $\gamma_{\varepsilon}=\tilde{a}|\log \varepsilon|$, where $\tilde{a}$ is a positive constant that will be fixed later. Observe that

$$
\frac{\gamma_{\varepsilon}}{\beta_{\varepsilon}} \rightarrow 0 \quad \text { as } \varepsilon \rightarrow 0
$$


and, since (3.1)

$$
P_{v_{m}}^{(\varepsilon)}\left(\tau^{\varepsilon}<\gamma_{\varepsilon}\right) \leq c_{n} \varepsilon^{n}
$$

Also let $F_{\varepsilon}(t)=P_{v_{m}}^{(\varepsilon)}\left(\tau^{\varepsilon}>t \beta_{\varepsilon}\right)$ and

$$
h_{\varepsilon}(t, s)=\frac{F_{\varepsilon}(t+s)}{F_{\varepsilon}(s)}-F_{\varepsilon}(t) .
$$

Proposition 3.1. For any $n \geq 0$, there exists $c_{n}$ such that for $\varepsilon$ small enough it holds

$$
\sup _{s \geq 0, t \geq \frac{\gamma_{\varepsilon}}{\beta_{\varepsilon}}}\left|h_{\varepsilon}(t, s)\right| \leq c_{n} \varepsilon^{n}
$$

We shall prove this proposition in the next section.

This result will allow us to prove the following proposition.

Proposition 3.2. For any $n \geq 1$ there exists $c_{n}$ such that for any $\varepsilon$ small enough the following holds

$$
d_{L}\left(\frac{\tau^{\varepsilon}}{\beta_{\varepsilon}}, \mathcal{E}\left(\lambda_{\varepsilon}\right)\right) \leq c_{n} \varepsilon^{n},
$$

where $d_{L}(\cdot, \cdot)$ refers to the Levy metric between two random variables, that is,

$$
\begin{aligned}
d_{L}(X, Y)= & \inf \{\varrho>0: \\
& P(X>t+\varrho)-\varrho \leq P(Y>t) \leq P(X>t-\varrho)+\varrho, \forall t \in \mathbb{R}\},
\end{aligned}
$$

and $\mathcal{E}(\lambda)$ is a exponential random variable with mean $\lambda^{-1}$, recall that $\lambda_{\varepsilon}^{-1}=$ $\mathbb{E}_{v_{m}}^{(\varepsilon)} \frac{\tau^{\varepsilon}}{\beta_{\varepsilon}}$.

We defer the proof of this proposition to the end of this section.

Finally, the Theorem 2.1 will be proved after showing that $\lambda_{\varepsilon} \rightarrow 1$, since this implies that $d_{L}\left(\mathcal{E}(1), \mathcal{E}\left(\lambda_{\varepsilon}\right)\right) \rightarrow 0$, and then

$$
d_{L}\left(\frac{\tau^{\varepsilon}}{\beta_{\varepsilon}}, \mathcal{E}(1)\right) \rightarrow 0 .
$$

The proof that $\lambda_{\varepsilon} \rightarrow 1$ follows easily. We give some details of the proof of this result, we follow the exponential characterization from Azlarov and Volodin (1986). We have that $\frac{\tau^{\varepsilon}}{\beta_{\varepsilon}}$ has finite moments of all orders, in particular $\lambda_{\varepsilon}^{-1}=$ $\mathbb{E}_{v_{m}}^{(\varepsilon)} \frac{\tau^{\varepsilon}}{\beta_{\varepsilon}}<\infty$. By definition $P_{v_{m}}^{(\varepsilon)}\left(\tau^{\varepsilon}>\beta_{\varepsilon}\right)=e^{-1}$, and this implies that $e^{-1} \leq \lambda_{\varepsilon}^{-1}$, so that $0<\lambda_{\varepsilon} \leq e$, for all $\varepsilon>0$.

Take any $\varrho \geq 0$ in the the defining property of $d_{L}\left(\frac{\tau^{\varepsilon}}{\beta_{\varepsilon}}, \mathcal{E}\left(\lambda_{\varepsilon}\right)\right)$ and $t=1$, so that:

$$
e^{-\lambda_{\varepsilon}(1+\varrho)}-\varrho \leq e^{-1} \leq e^{-\lambda_{\varepsilon}(1-\varrho)}+\varrho,
$$


then

$$
\begin{aligned}
e^{-1}-e^{-\lambda_{\varepsilon}} & \leq e^{-\lambda_{\varepsilon}}\left(e^{\lambda_{\varepsilon} \varrho}-1\right)+\varrho \\
& \leq \varrho\left(e^{\varrho \varrho+1}+1\right),
\end{aligned}
$$

also

$$
e^{-1}-e^{-\lambda_{\varepsilon}} \geq\left(e^{-e \varrho}-1\right)-\varrho \geq-\varrho\left(e^{1-e \varrho}+1\right),
$$

but the infimum of such $\varrho$ is less than $c_{n} \varepsilon^{n}$, so that

$$
\left|e^{-1}-e^{-\lambda_{\varepsilon}}\right| \leq c_{n}^{\prime} \varepsilon^{n},
$$

and then $\lambda_{\varepsilon} \rightarrow 1$, as $\varepsilon$ goes to zero.

Proof of Proposition 3.2. It's enough to prove that the two inequalities in the definition of the Levy distance, $d_{L}\left(\frac{\tau^{\varepsilon}}{\beta_{\varepsilon}}, \mathcal{E}\left(\lambda_{\varepsilon}\right)\right)$, hold for $\varrho=c_{n} \varepsilon^{n}$, with $n \geq 1$.

Take $\theta_{\varepsilon}(t)=\lambda_{\varepsilon} \int_{0}^{\infty} P_{v_{m}}^{(\varepsilon)}\left(\tau^{\varepsilon}>s \beta_{\varepsilon}\right) h_{\varepsilon}(t, s) d s$, note that $\left|\theta_{\varepsilon}(t)\right| \leq 1$, for all $t$, and by Proposition 3.1, for $t \geq \frac{\gamma_{\varepsilon}}{\beta_{\varepsilon}},\left|\theta_{\varepsilon}(t)\right| \leq c_{n} \varepsilon^{n}$. From Azlarov and Volodin (1986), the following representation of $F_{\varepsilon}(t)$ holds:

$$
F_{\varepsilon}(t)=e^{-\lambda_{\varepsilon} t}+\lambda_{\varepsilon} \int_{0}^{t} e^{-\lambda_{\varepsilon}(t-u)} \theta_{\varepsilon}(u) d u-\theta_{\varepsilon}(t) .
$$

We begin with the first inequality of the Levy metric. Since, for $k>n$ large and $\varepsilon$ small enough $\frac{\gamma_{\varepsilon}}{2 c_{n} \varepsilon^{n}} \leq \varepsilon^{-k}<\beta_{\varepsilon}$, then $\frac{\gamma_{\varepsilon}}{\beta_{\varepsilon}} \ll 2 c_{n} \varepsilon^{n}=\varrho$, So that $t+\varrho>\frac{\gamma_{\varepsilon}}{\beta_{\varepsilon}}$, and we can use the Proposition 3.1 to get

$$
\begin{aligned}
F_{\varepsilon}(t+\varrho)= & e^{-\lambda_{\varepsilon}(t+\varrho)}+\lambda_{\varepsilon} \int_{0}^{(t+\varrho)} e^{-\lambda_{\varepsilon}(t+\varrho-u)} \theta_{\varepsilon}(u) d u-\theta_{\varepsilon}(t+\varrho) \\
\leq & e^{-\lambda_{\varepsilon}(t+\varrho)}+\lambda_{\varepsilon} \int_{0}^{\frac{\gamma_{\varepsilon}}{\beta_{\varepsilon}}} e^{-\lambda_{\varepsilon}(t+\varrho-u)} d u \\
& +c_{n} \varepsilon^{n} \lambda_{\varepsilon} \int_{\frac{\gamma_{\varepsilon}}{\beta_{\varepsilon}}}^{(t+\varrho)} e^{-\lambda_{\varepsilon}(t+\varrho-u)} d u+c_{n} \varepsilon^{n} \\
\leq & e^{-\lambda_{\varepsilon} t} e^{-\lambda_{\varepsilon}\left(\varrho-\frac{\gamma_{\varepsilon}}{\beta_{\varepsilon}}\right)}+\varrho \\
\leq & e^{-\lambda_{\varepsilon} t}+\varrho .
\end{aligned}
$$

Now for the other inequality in the Levy metric, we must analyse various cases

(i) If $t<\varrho$, then $F_{\varepsilon}(t-\varrho)=1$ so obviously $F_{\varepsilon}(t-\varrho) \geq e^{-\lambda_{\varepsilon} t}-\varrho$.

(ii) If $t>\varrho$ and $t-\varrho<\frac{\gamma_{\varepsilon}}{\beta_{\varepsilon}}$ then $F_{\varepsilon}(t-\varrho) \geq F_{\varepsilon}\left(\frac{\gamma_{\varepsilon}}{\beta_{\varepsilon}}\right) \geq 1-c_{n} \varepsilon^{n} \geq e^{-\lambda_{\varepsilon} t}-\varrho$, by Eq. (3.3). 
(iii) If $t>\varrho$ and $t-\varrho>\frac{\gamma_{\varepsilon}}{\beta_{\varepsilon}}$ then

$$
\begin{aligned}
F_{\varepsilon}(t-\varrho)= & e^{-\lambda_{\varepsilon}(t-\varrho)}+\lambda_{\varepsilon} \int_{0}^{(t-\varrho)} e^{-\lambda_{\varepsilon}(t-\varrho-u)} \theta_{\varepsilon}(u) d u-\theta_{\varepsilon}(t-\varrho) \\
\geq & e^{-\lambda_{\varepsilon}(t-\varrho)}-\lambda_{\varepsilon} \int_{0}^{\frac{\gamma_{\varepsilon}}{\beta_{\varepsilon}}} e^{-\lambda_{\varepsilon}(t-\varrho-u)} d u \\
& -c_{n} \varepsilon^{n} \lambda_{\varepsilon} \int_{\frac{\gamma_{\varepsilon}}{\beta_{\varepsilon}}}^{(t+\varrho)} e^{-\lambda_{\varepsilon}(t+\varrho-u)} d u-c_{n} \varepsilon^{n} \\
\geq & e^{-\lambda_{\varepsilon}(t-\varrho)}-\left(e^{-\lambda_{\varepsilon}\left(t-\varrho-\frac{\gamma_{\varepsilon}}{\beta_{\varepsilon}}\right)}-e^{-\lambda_{\varepsilon}(t-\varrho)}\right)-\varrho \\
= & e^{-\lambda_{\varepsilon} t}\left[2 e^{\lambda_{\varepsilon} \varrho}-e^{\lambda_{\varepsilon}\left(\varrho+\frac{\gamma_{\varepsilon}}{\beta_{\varepsilon}}\right)}\right]-\varrho .
\end{aligned}
$$

All we need to prove is that $2 e^{\lambda_{\varepsilon} \varrho}-e^{\lambda_{\varepsilon}\left(\varrho+\frac{\gamma_{\varepsilon}}{\beta_{\varepsilon}}\right)}>1$. To this end, first observe that for $z$ close to 1 we have that $2 z-1>z^{3 / 2}$. Since $\varrho=2 c_{n} \varepsilon^{n}$ and $0 \leq \lambda_{\varepsilon} \leq e$ we have that $\lambda_{\varepsilon} \varrho \rightarrow 0$ as $\varepsilon \rightarrow 0$, so that taking $z=e^{\lambda_{\varepsilon} \varrho}$, for $\varepsilon$ small enough,

$$
2 e^{\lambda_{\varepsilon} \varrho}-e^{3 / 2 \lambda_{\varepsilon} \varrho}>1 \text {. }
$$

But $\frac{\gamma_{\varepsilon}}{\beta_{\varepsilon}}<\varrho / 2$, so $e^{\lambda_{\varepsilon}\left(\varrho+\frac{\gamma_{\varepsilon}}{\beta_{\varepsilon}}\right)}<e^{3 / 2 \lambda_{\varepsilon} \varrho}$, using this in (3.5), we can conclude that

$$
F_{\varepsilon}(t-\varrho) \geq e^{-\lambda_{\varepsilon} t}-\varrho
$$

So, the infimum in the Levy distance, is less than $\varrho=c_{n} \varepsilon^{n}$, and the proposition is proved.

\section{Loss of memory property}

In this section we prove the loss of memory property of $\tau^{\varepsilon}$.

Proof of Proposition 3.1. We shall prove that

$$
\begin{aligned}
& \left|P_{v_{m}}^{(\varepsilon)}\left(\tau^{\varepsilon}>(t+s) \beta_{\varepsilon}\right)-P_{v_{m}}^{(\varepsilon)}\left(\tau^{\varepsilon}>t \beta_{\varepsilon}\right) P_{v_{m}}^{(\varepsilon)}\left(\tau^{\varepsilon}>s \beta_{\varepsilon}\right)\right| \\
& \quad \leq c_{n} \varepsilon^{n} P_{v_{m}}^{(\varepsilon)}\left(\tau^{\varepsilon}>s \beta_{\varepsilon}\right),
\end{aligned}
$$

for $t \geq \frac{\gamma_{\varepsilon}}{\beta_{\varepsilon}}$. To do this, we shall condition on the first summand at time $s \beta_{\varepsilon}+\gamma_{\varepsilon}$, and after some algebra we will get the difference between terms like $P_{\sigma}^{(\varepsilon)}\left(\tau^{\varepsilon}>t \beta_{\varepsilon}\right)$, $\sigma \in A_{\delta^{\prime}}^{(\varepsilon)}$, multiplied by $P_{v_{m}}^{(\varepsilon)}\left(\tau^{\varepsilon}>s \beta_{\varepsilon}\right)$, with $0<\delta^{\prime}<\delta$. So, we need to bound such differences. The main tool is the following proposition, that permits us to couple two processes before the exit time of $A_{\delta}^{(\varepsilon)}$. Recall that $\gamma_{\varepsilon}=\tilde{a}|\log \varepsilon|$. 
Proposition 4.1. Let $0<\delta^{\prime}<\delta$. For any $n \geq 0$ there exists $c_{n}>0$ such that for any $\varepsilon>0$ small enough, the following holds:

$$
\begin{aligned}
\sup _{t \geq 0} \mid & P_{\sigma}^{(\varepsilon)}\left(\tau^{\varepsilon}>t \beta_{\varepsilon}\right)-P_{\sigma^{\prime}}^{(\varepsilon)}\left(\tau^{\varepsilon}>t \beta_{\varepsilon}\right) \mid \\
& \leq P_{\sigma}^{(\varepsilon)}\left(\tau^{\varepsilon}<\gamma_{\varepsilon}\right)+P_{\sigma^{\prime}}^{(\varepsilon)}\left(\tau^{\varepsilon}<\gamma_{\varepsilon}\right)+c_{n} \varepsilon^{n},
\end{aligned}
$$

for any $\sigma, \sigma^{\prime} \in A_{\delta^{\prime}}^{(\varepsilon)}$.

We defer the proof to the end of the section. Turning to the proof of (4.1), note that $\gamma_{\varepsilon}<\beta_{\varepsilon}$ and for $n \geq 1$ there exists $c_{n}>0$ such that

$$
P_{v_{m}}^{(\varepsilon)}\left(\tau^{\varepsilon}<\gamma_{\varepsilon}\right) \leq c_{n} \varepsilon^{n},
$$

for $\varepsilon$ small enough. We begin by bounding from above

$$
P_{v_{m}}^{(\varepsilon)}\left(\tau^{\varepsilon}>(t+s) \beta_{\varepsilon}\right)-P_{v_{m}}^{(\varepsilon)}\left(\tau^{\varepsilon}>t \beta_{\varepsilon}\right) P_{v_{m}}^{(\varepsilon)}\left(\tau^{\varepsilon}>s \beta_{\varepsilon}\right) .
$$

Since, by hypothesis, $t \beta_{\varepsilon} \geq \gamma_{\varepsilon}$, we can condition the process up to time $s \beta_{\varepsilon}+\gamma_{\varepsilon}$ and after using the Markov property, we get that this expression is less than

$$
\begin{aligned}
& \mathbb{E}_{v_{m}}^{(\varepsilon)}\left(\mathbf{1}_{\tau^{\varepsilon}>s \beta_{\varepsilon}} P_{\sigma_{s} \beta_{\varepsilon}+\gamma_{\varepsilon}}^{(\varepsilon)}\left(\tau^{\varepsilon}>t \beta_{\varepsilon}-\gamma_{\varepsilon}\right)\right)-P_{v_{m}}^{(\varepsilon)}\left(\tau^{\varepsilon}>t \beta_{\varepsilon}-\gamma_{\varepsilon}\right) P_{v_{m}}^{(\varepsilon)}\left(\tau^{\varepsilon}>s \beta_{\varepsilon}\right) \\
& \quad+P_{v_{m}}^{(\varepsilon)}\left(\tau^{\varepsilon}>t \beta_{\varepsilon}-\gamma_{\varepsilon}\right) P_{v_{m}}^{(\varepsilon)}\left(\tau^{\varepsilon}>s \beta_{\varepsilon}\right)-P_{v_{m}}^{(\varepsilon)}\left(\tau^{\varepsilon}>t \beta_{\varepsilon}\right) P_{v_{m}}^{(\varepsilon)}\left(\tau^{\varepsilon}>s \beta_{\varepsilon}\right) .
\end{aligned}
$$

Decomposing the first summand according to $\left\{\sigma_{s} \beta_{\varepsilon}+\gamma_{\varepsilon} \in A_{\delta^{\prime}}\right\}$, or not (recall that $\delta^{\prime}<\delta$ ), the last expression is

$$
\begin{aligned}
\mathbb{E}_{v_{m}}^{(\varepsilon)}\left(\mathbf{1}_{\tau^{\varepsilon}>s \beta_{\varepsilon}} \mathbf{1}_{\sigma_{s} \beta_{\varepsilon}+\gamma_{\varepsilon}} \in A_{\delta^{\prime}}\right. & {\left.\left[P_{\sigma_{s} \beta_{\varepsilon}+\gamma_{\varepsilon}}^{(\varepsilon)}\left(\tau^{\varepsilon}>t \beta_{\varepsilon}-\gamma_{\varepsilon}\right)-P_{v_{m}}^{(\varepsilon)}\left(\tau^{\varepsilon}>t \beta_{\varepsilon}-\gamma_{\varepsilon}\right)\right]\right) } \\
& +\mathbb{E}_{v_{m}}^{(\varepsilon)}\left(\mathbf{1}_{\tau^{\varepsilon}>s \beta_{\varepsilon}} \mathbf{1}_{\sigma_{s \beta_{\varepsilon}+\gamma_{\varepsilon}} \notin A_{\delta^{\prime}}}\left[P_{\sigma_{s} \beta_{\varepsilon}+\gamma_{\varepsilon}}^{(\varepsilon)}\left(\tau^{\varepsilon}>t \beta_{\varepsilon}-\gamma_{\varepsilon}\right)-P_{v_{m}}^{(\varepsilon)}\left(\tau^{\varepsilon}>t \beta_{\varepsilon}-\gamma_{\varepsilon}\right)\right]\right) \\
& +P_{v_{m}}^{(\varepsilon)}\left(\tau^{\varepsilon}>s \beta_{\varepsilon}\right)\left[P_{v_{m}}^{(\varepsilon)}\left(\tau^{\varepsilon}>t \beta_{\varepsilon}-\gamma_{\varepsilon}\right)-P_{v_{m}}^{(\varepsilon)}\left(\tau^{\varepsilon}>t \beta_{\varepsilon}\right)\right] .
\end{aligned}
$$

By Proposition 4.1, the first term in (4.3) can be bounded by:

$$
\mathbb{E}_{v_{m}}^{(\varepsilon)}\left(\mathbf{1}_{\tau^{\varepsilon}>s \beta_{\varepsilon}} \mathbf{1}_{\sigma_{s} \beta_{\varepsilon}+\gamma_{\varepsilon} \in A_{\delta^{\prime}}} P_{\sigma_{s} \beta_{\varepsilon}+\gamma_{\varepsilon}}^{(\varepsilon)}\left(\tau^{\varepsilon} \leq \gamma_{\varepsilon}\right)\right)+c_{n} \varepsilon^{n} P_{v_{m}}^{(\varepsilon)}\left(\tau^{\varepsilon}>s \beta_{\varepsilon}\right) .
$$

The first summand could be expressed, after conditioning up to $s \beta_{\varepsilon}+\frac{\gamma_{\varepsilon}}{2}$, as

$$
\begin{aligned}
& \mathbb{E}_{v_{m}}^{(\varepsilon)}\left(\mathbf{1}_{\tau^{\varepsilon}>s \beta_{\varepsilon}} \mathbf{1}_{\sigma_{s \beta_{\varepsilon}+\frac{\gamma_{\varepsilon}}{2}} \in A_{\delta^{\prime}}} P_{\sigma_{s \beta_{\varepsilon}+\frac{\gamma_{\varepsilon}}{2}}^{(\varepsilon)}}\left(\exists u, \frac{\gamma_{\varepsilon}}{2} \leq u \leq \frac{3 \gamma_{\varepsilon}}{2}, \sigma_{u} \notin A_{\delta}\right)\right) \\
& \quad+c_{n} \varepsilon^{n} P_{v_{m}}^{(\varepsilon)}\left(\tau^{\varepsilon}>s \beta_{\varepsilon}\right) .
\end{aligned}
$$

The conditional probability that appears inside, could be bounded by $c_{n} \varepsilon^{n}$, since if $\sigma \in A_{\delta}$ then, at times of order $\gamma_{\varepsilon}$, it belongs to $A_{\delta^{\prime}}, \delta>\delta^{\prime}$ with probability of order $c_{n} \varepsilon^{n}$. For the proof of this we can follow the same steps of Proposition 4.3 in Hinojosa (2004). 
So that the first summand in (4.3) can be bounded by

$$
P_{v_{m}}^{(\varepsilon)}\left(\tau^{\varepsilon}>s \beta_{\varepsilon}\right) c_{n} \varepsilon^{n} .
$$

In similar way for the second term of (4.3), after conditioning up to time $s \beta_{\varepsilon}$ :

$$
\begin{aligned}
2 \mathbb{E}_{v_{m}}^{(\varepsilon)}\left(\mathbf{1}_{\tau^{\varepsilon}>s \beta_{\varepsilon}} \mathbf{1}_{\sigma_{s \beta_{\varepsilon}+\gamma_{\varepsilon}} \notin A_{\delta^{\prime}}}\right) & \leq 2 \mathbb{E}_{v_{m}}^{(\varepsilon)}\left(\mathbf{1}_{\tau^{\varepsilon}>s \beta_{\varepsilon}} P_{\sigma_{s} \beta_{\varepsilon}}^{(\varepsilon)}\left(\sigma_{\gamma_{\varepsilon}} \notin A_{\delta^{\prime}}\right)\right) \\
& \leq 2 P_{v_{m}}^{(\varepsilon)}\left(\tau^{\varepsilon}>s \beta_{\varepsilon}\right) c_{n} \varepsilon^{n},
\end{aligned}
$$

where we used again Hinojosa (2004), in the second inequality.

Finally, the third term in (4.3), after decomposing according to the event $\left\{\sigma_{\gamma_{\varepsilon}} \in A_{\delta^{\prime}}\right\}$, and using the Proposition 4.1. As above, we get the same bound:

$$
\begin{aligned}
P_{v_{m}}^{(\varepsilon)}\left(\tau^{\varepsilon}>s \beta_{\varepsilon}\right)\left(P_{v_{m}}^{(\varepsilon)}\left(\tau^{\varepsilon}>t \beta_{\varepsilon}-\gamma_{\varepsilon}\right)-\mathbb{E}_{v_{m}}^{(\varepsilon)}\left(\mathbf{1}_{\tau^{\varepsilon}>\gamma_{\varepsilon}} P_{\sigma_{\gamma_{\varepsilon}}}^{(\varepsilon)}\left(\tau^{\varepsilon}>t \beta_{\varepsilon}-\gamma_{\varepsilon}\right)\right)\right) \\
\leq P_{v_{m}}^{(\varepsilon)}\left(\tau^{\varepsilon}>s \beta_{\varepsilon}\right) \\
\quad \times\left(2 P_{v_{m}}^{(\varepsilon)}\left(\tau^{\varepsilon} \leq \gamma_{\varepsilon}\right)+c_{n} \varepsilon^{n}+\mathbb{E}_{v_{m}}^{(\varepsilon)}\left(\mathbf{1}_{\sigma_{\gamma_{\varepsilon}} \in A_{\delta^{\prime}}} P_{\sigma_{\gamma_{\varepsilon}}(\varepsilon)}\left(\tau^{\varepsilon} \leq \gamma_{\varepsilon}\right)\right)\right) \\
\leq P_{v_{m_{-}}}^{(\varepsilon)}\left(\tau^{\varepsilon}>s \beta_{\varepsilon}\right) c_{n} \varepsilon^{n} .
\end{aligned}
$$

So, until now we proved that

$$
P_{v_{m}}^{(\varepsilon)}\left(\tau^{\varepsilon}>(t+s) \beta_{\varepsilon}\right)-P_{v_{m}}^{(\varepsilon)}\left(\tau^{\varepsilon}>t \beta_{\varepsilon}\right) P_{v_{m}}^{(\varepsilon)}\left(\tau^{\varepsilon}>s \beta_{\varepsilon}\right)
$$

is bounded from above by $P_{v_{m}}^{(\varepsilon)}\left(\tau^{\varepsilon}>s \beta_{\varepsilon}\right) c_{n} \varepsilon^{n}$, for $s>0$ and $t>\frac{\gamma_{\varepsilon}}{\beta_{\varepsilon}}$. Now, proceeding similarly we shall get the same bound by below, and the proposition is proved.

Proof of Proposition 4.1. Note that, if we take $\sigma, \sigma^{\prime} \in A_{\delta^{\prime}}^{(\varepsilon)}$, and consider the coupling $P_{\sigma, \sigma^{\prime}}^{(\varepsilon)}$ of two process starting at $\sigma, \sigma^{\prime}$, see the Appendix below, then:

$$
\begin{aligned}
\mid P_{\sigma}^{(\varepsilon)}( & \left.\tau_{\sigma}^{\varepsilon}>t \beta_{\varepsilon}\right)-P_{\sigma^{\prime}}^{(\varepsilon)}\left(\tau_{\sigma^{\prime}}^{\varepsilon}>t \beta_{\varepsilon}\right) \mid \\
\leq & P_{\sigma, \sigma^{\prime}}^{(\varepsilon)}\left(\tau_{\sigma}^{\varepsilon}>t \beta_{\varepsilon}, \tau_{\sigma^{\prime}}^{\varepsilon} \leq t \beta_{\varepsilon}\right)+P_{\sigma, \sigma^{\prime}}^{(\varepsilon)}\left(\tau_{\sigma^{\prime}}^{\varepsilon}>t \beta_{\varepsilon}, \tau_{\sigma}^{\varepsilon} \leq t \beta_{\varepsilon}\right) \\
\leq & P_{\sigma, \sigma^{\prime}}^{(\varepsilon)}\left(\tau_{\sigma}^{\varepsilon}>t \beta_{\varepsilon}, \tau_{\sigma^{\prime}}^{\varepsilon}<\gamma_{\varepsilon}\right)+P_{\sigma, \sigma^{\prime}}^{(\varepsilon)}\left(\tau_{\sigma}^{\varepsilon}>t \beta_{\varepsilon}, \gamma_{\varepsilon} \leq \tau_{\sigma^{\prime}}^{\varepsilon} \leq t \beta_{\varepsilon}\right) \\
& \quad+P_{\sigma, \sigma^{\prime}}^{(\varepsilon)}\left(\tau_{\sigma^{\prime}}^{\varepsilon}>t \beta_{\varepsilon}, \tau_{\sigma}^{\varepsilon}<\gamma_{\varepsilon}\right)+P_{\sigma, \sigma^{\prime}}^{(\varepsilon)}\left(\tau_{\sigma^{\prime}}^{\varepsilon}>t \beta_{\varepsilon}, \gamma_{\varepsilon} \leq \tau_{\sigma}^{\varepsilon} \leq t \beta_{\varepsilon}\right) \\
\leq & P_{\sigma}^{(\varepsilon)}\left(\tau^{\varepsilon}<\gamma_{\varepsilon}\right)+P_{\sigma^{\prime}}^{(\varepsilon)}\left(\tau^{\varepsilon}<\gamma_{\varepsilon}\right)+2 P_{\sigma, \sigma^{\prime}}^{(\varepsilon)}\left(\sigma_{\gamma_{\varepsilon}} \neq \sigma_{\gamma_{\varepsilon}}^{\prime}\right) .
\end{aligned}
$$

For the last term, we have

$$
\begin{aligned}
P_{\sigma, \sigma^{\prime}}^{(\varepsilon)}\left(\sigma_{\gamma_{\varepsilon}} \neq \sigma_{\gamma_{\varepsilon}}^{\prime}\right) & \leq \sum_{x \in \mathbb{Z}_{\varepsilon}} P_{\sigma, \sigma^{\prime}}^{(\varepsilon)}\left(\sigma_{\gamma_{\varepsilon}}(x) \neq \sigma_{\gamma_{\varepsilon}}^{\prime}(x)\right) \\
& \leq \varepsilon^{-1} \mathbb{E}_{\sigma, \sigma^{\prime}}^{(\varepsilon)}\left|\sigma_{\gamma_{\varepsilon}}(0)-\sigma_{\gamma_{\varepsilon}}^{\prime}(0)\right|
\end{aligned}
$$

in the last inequality by translation invariance. 
Thus, the main step of the proof is to get a bound for the last term. For this part we use Theorem 2.1 in Brassesco et al. (2000a), as stated below, where it is found a bound of order $\varepsilon^{n}$ for the discrepancies between the processes $\sigma_{T}^{+}$and $\sigma_{T}^{-}$, which is defined as the expected value of the site differences for the coupling $P_{\mathbf{1},-\mathbf{1}}^{(\varepsilon)}$ of the processes that starts from $\mathbf{1}$ and $\mathbf{- 1}$ and at times $T:=\tilde{a}|\ln \varepsilon|$ : $\mathbb{E}_{\mathbf{1},-\mathbf{1}}^{(\varepsilon)}\left(\sigma_{T}^{+}(0)-\sigma_{T}^{-}(0)\right)$. Using this result and that the coupling is order preserving, see the Appendix, we have the following proposition.

Proposition 4.2. For any $\gamma<\frac{1}{2}, n \geq 1$ and $\varepsilon>0$ small enough there exists positive constants $\tilde{a}$ and $c_{n}$ such that:

$$
0 \leq \mathbb{E}_{\sigma, \sigma^{\prime}}^{(\varepsilon)}\left|\sigma_{T}(0)-\sigma_{T}^{\prime}(0)\right| \leq c_{n} \varepsilon^{n},
$$

where $T:=\tilde{a}|\ln \varepsilon|$, and $\sigma, \sigma^{\prime} \in A_{\delta}$.

Observe that by attractiveness of the coupling at time $T: \sigma_{T}^{+}(x) \geq \sigma_{T}(x)$ and $\sigma_{T}(x) \geq \sigma_{T}^{-}(x)$ for any $x \in \mathbb{Z}_{\varepsilon}$ and any processes $\sigma_{t}$.

Using this result, the right-hand side of (4.8) could be bounded by $c_{n} \varepsilon^{n-1}$ uniformly in $A_{\delta}$, and the Proposition 4.1 is proved.

\section{Appendix: Graphical representation}

In this section, we will describe the graphical construction of the process, due to Harris (see Haken (1978)), the same that appears in Brassesco et al. (2000b), see also Brassesco et al. (2000a). This allows us to exploit the comparison between different evolutions of the process.

For each $x \in \mathbb{Z}_{\varepsilon}$ let $\mathcal{N}^{x,+}, \mathcal{N}^{x,-}$, be Poisson process of intensity $c_{\max }:=$ $(1+\gamma)^{2}+\frac{\mu}{2}$ (i.e., the maximum flip rate for the Glauber process), and for each bond $\left(x, x+e_{i}\right)$ (where $e_{i}=(0, \ldots, 1, \ldots, 0)$, one in the $i$ th position), let $\mathcal{N}_{x, e_{i}}^{\varepsilon}$ be a Poisson process of intensity $\varepsilon^{-2}$. Let also $U_{n}^{x,+}, U_{n}^{x,-}, n \geq 1$ be i.i.d. Uniform $(0,1)$ random variables. We also assume that all the process $\mathcal{N}^{x,+}, \mathcal{N}^{x,-}, \mathcal{N}_{x, e_{i}}^{\varepsilon}$ and $\left\{U_{n}^{x,+}\right\}_{n \geq 1},\left\{U_{n}^{x,-}\right\}_{n \geq 1}, n \geq 1, x \in \mathbb{Z}_{\varepsilon}$ are independent of each other.

The graphical representation is a realization of the process which applies for any initial configuration. Whenever a mark $\mathcal{N}_{x, e_{i}}^{\varepsilon}$ appears then we exchange the spins at $x$ and $x+e_{i}$. At the time of the $n$th mark of $\mathcal{N}^{x,+}\left(\mathcal{N}^{x,-}\right)$, we flip the spin at $x$ if $\sigma(x)=1$ and $c_{\mu}(x, \sigma)>U_{n}^{x,+} c_{\max }\left(\sigma(x)=-1\right.$ and $\left.c_{\mu}(x, \sigma)>U_{n}^{x,-} c_{\max }\right)$. This prescription defines the evolution of the Glauber + Kawasaki process.

In the marked process, consider a realization $\omega$. For each $x \in \mathbb{Z}_{\varepsilon}$, and $t \geq 0$, there are functions $f_{x, t}(\sigma, \omega)$ with the property that, once $\sigma$ is fixed, the law of the variables $\sigma_{t}(x):=f_{x, t}(\sigma, \omega)$, is the same as the law of the Glauber + Kawasaki process which starts from $\sigma$; moreover, $f_{x, t}(\sigma, \omega) \leq f_{x, t}\left(\sigma^{\prime}, \omega\right)$ if $\sigma \leq \sigma^{\prime}$. This coupling preserves the order since the rates are attractive. 


\title{
Acknowledgments
}

Part of this work was done at Instituto de Matemática Pura e Aplicada with help of a CNPq Grant 142919.

This work is a partial consequence of the author's Ph.D. thesis, who would like to thank his adviser, professor M. E. Vares, for presenting the problem and contributing with valuable comments and suggestions.

\section{References}

Azlarov, T. A. and Volodin, N. A. (1986). Characterization Problems Associated with the Exponential Distribution. New York: Springer. MR0841073

Brassesco, S., Olivieri, E. and Vares, M. E. (1998). Couplings and asymptotic exponentiality of exit times. Journal of Statistical Physics 93, 393-404. MR1656375

Brassesco, S., Presutti, E., Sidoravicius, V. and Vares, M. E. (2000a). Ergodicity and exponential convergence of a Glauber + Kawasaki process. American Mathematical Society Translations, Series 2 198, 37-49. MR1766341

Brassesco, S., Presutti, E., Sidoravicius, V. and Vares, M. E. (2000b). Ergodicity of a Glauber + Kawasaki process with metastable states. Markov Processes and Related Fields 6, 181-203. MR1778754

De Masi, A., Ferrari, P. A. and Lebowitz, J. L. (1986). Reaction-diffusion equations for interacting particle systems. Journal of Statistical Physics 44, 589-644. MR0857069

De Masi, A. and Presutti, E. (1991). Mathematical Methods for Hydrodynamic Limits. Lecture Notes in Mathematics 1501. Berlin: Springer. MR1175626

Durrett, R. and Neuhauser, C. (1994). Particle systems and reaction-diffusion equations. Annals of Probability 22, 289-333. MR1258879

Faris, W. and Jona-Lasinio, G. (1982). Large fluctuations for a nonlinear heat equation with noise. Journal of Physics A, Mathematical and General 15, 3025-3055. MR0684578

Haken, H. (1978). Synergetics-A field beyond irreversible thermodynamics. In Stochastic Processes in Nonequilibrium Systems. Lecture Notes in Physics 84, 139-167. MR0532362

Hinojosa, A. (2004). Exit time for a reaction diffusion model. Markov Processes and Related Fields 10, 705-744. MR2127751

Martinelli, F., Olivieri, E. and Scoppola, E. (1989). Small random perturbations of finite- and infinitedimensional dynamical systems: Unpredictability of exit times. Journal of Statistical Physics $\mathbf{5 5}$, 477-504. MR1003525

\author{
Statistics Department \\ Federal University of Minas Gerais \\ Av. Antônio Carlos, 6627, \\ Pampulha-CEP 31270-901 \\ Belo Horizonte \\ Brazil \\ E-mail: adrian@est.ufmg.br
}

